\title{
Thank You Note to all our 2021 Reviewers
}

Published online: 8 March 2022

(c) Springer Science+Business Media, LLC, part of Springer Nature 2022

The Editors-in-Chief of Marine Biotechnology are very grateful to all authors, reviewers, and Editorial Board members who dedicated their time and expertise to help sustain the editorial quality of the journal in 2021. Although the COVID 19 pandemic created personal and professional hardships for everyone these past two years, we are especially grateful for the devotion shown by our esteemed peer reviewers who continue to give of their time and share their scientific knowledge despite these very difficult circumstances.

A heartfelt "Thank You" to all of our 2021 peer reviewers!

\begin{tabular}{|c|c|c|}
\hline Roberto T. Abdala Díaz & C Bouza & Gary Dickinson \\
\hline Amal Abdelhamid & Elena Bovio & Shaoxiong Ding \\
\hline Jason Abernathy & Jean-François Briand & Thuoc Doan Van \\
\hline Vojtech Adam & Chiara Bulfon & Sergey Dobretsov \\
\hline Yasuo Agawa & Alejandro Buschmann & Jose Domingos \\
\hline Sylvain Agostini & Gary Caldwell & Shaojun (Jim) Du \\
\hline Qinhui Ai & Huansheng $\mathrm{Cao}$ & Xiaodong Du \\
\hline Tsunehiro Aki & Mingfeng Cao & Yifeng Du \\
\hline Nick Aldred & Florian Cazals & Cunming Duan \\
\hline Nick Aldred & Serap Celikler & Laurent Dufossé \\
\hline Rosa Alduina & Ching-Fong Chang & Sylvie Dufour \\
\hline Rafet Al-Tobasei & José Cheel & Alain Dufour \\
\hline Heidi Amlund & Shaoxing Chen & Justine Dumay \\
\hline Hirokazu Aoki & Ting Chen & Rex Dunham \\
\hline Hiroya Araie & Xianzhong Chen & Michael Dunn \\
\hline Atsushi Arakaki & Songlin Chen & Catherine Dupré \\
\hline Osamu Arakawa & Man Kit Cheung & Hjorleifur Einarsson \\
\hline Sylvain Aubry & Heng Chi & Jindarat Ekprasert \\
\hline Amr Badary & Ka-Hou Chu & Marek Elias \\
\hline Zhiyi Bai & Thavasimuthu Citarasu & Abigail Elizur \\
\hline Ranjith Kumar Bakku & Beth Cleveland & Hirotoshi Endo \\
\hline Lisui Bao & Jackie L. Collier & Kamil Eryalçın \\
\hline Baolong Bao & Sheelagh Conlan & Manuel Espinosa \\
\hline Brian Beckman & Gérald Culioli & Yuding Fan \\
\hline Kirsten Benkendorff & Leonardo Curatti & Mohammad Ali Faramarzi \\
\hline Sophie Berland & Asher Cutter & Mark Fast \\
\hline Peggy Biga & Roy Danzmann & Fabienne Fay \\
\hline Jamie Bojko & Denis de la Broise & Dandan Feng \\
\hline Isabelle Bonnard & Christine Delbarre-Ladrat & Danqing Feng \\
\hline Debajit Borah & Paul Deleris & Pedro Fernandes \\
\hline Nathalie Bourgougnon & Andrew Desbois & Ignacio Fernández \\
\hline Marie-Lise Bourguet-Kondracki & Raunak Dhanker & Catalina Fernández-Díaz \\
\hline
\end{tabular}


Roberto Fernandez-Lafuente

Roberto Feuda

Peter Fields

Garth Fletcher

Jose David Flores

Jose Luis Fonseca

Valentin Foulon

Warren Francis

Shoko Fujiwara

Yuhua Gao

Zexia Gao

Kunshan Gao

Jian Gao

Uri Gat

Rick Goetz

Olivier Goncalves

Zhiyuan Gong

Yoav Gothilf

Dominique Grizeau

Joshua Gross

Baocheng Guo

Huarong Guo

Ximing Guo

Margarita Gutiérrez

Perry Hackett

Kyoko Hagino-Tomioka

Eric Hallerman

Bojan Hamer

Ziqiang Han

Espen Hansen

Hirofumi Hara

Ronald Hardy

Mate Hare

Ben Harvey

Yuji Hatada

Kyoko Hayashi

Masahiro Hayashi

Tonje Heggeset

Claire Hellio

Yukako Hihara

Jun-ichi Hikima

Ikuo Hirono

Saito Hiroshi

Corey Holt

Ross Houston

Wei Hu

Xiaoli $\mathrm{Hu}$

Yue Gen Hua

H. Huang

Ming-Chih Huang

Wenshu Huang

Xiaoting Huang

Zhijian Huang
Angela Iannitelli

Kazuho Ikeo

Yoshio Ikezawa

Chiaki Imada

Kazuo Inoue

Akira Inoue

Shoichiro Ishizaki

Makoto Ito

Koji Iwamoto

Harini Iyer

Mohamed Jebbar

Xiangshan Ji

EonSeon Jin

Alyssa Joyce

Naoki Kabeya

Masataka Kajikawa

Ryoma Kamikawa

Kei Kamino

Mitsunobu Kamiya

Gen Kaneko

John Kavanagh

Shigeyuki Kawai

Sunil Khare

KT Kim

Se-Kwon Kim

Yoshikazu Kitano

Alexa Klettner

Venkata Kokati

Hidehiro Kondo

Emily Kostas

Robert Kourist

Takashi Koyama

Chin-Long KY

Antje Labes

Joao Henrique Lago

Chris Langdon

Gwenaelle Le Blay

Laurent Lebrun

Ching-Fu Lee

Tse-Min Lee

Florian Lelchat

Orly Levitan

Oshrat Levy-Ontman

Chao Li

Chenghua Li

David Li

Erchao Li

Fuhua Li

Fu-Li Li

Jianzhen Li

Mingyou Li

Ning Li

Qi Li
Shihao Li

Yun Li

Zhaoxia Li

Zhongjie Li

Jiale Li

Yuanyou Li

Fuchuan Li

Ana Isabel Lillebo

Leongseng Lim

Chun-Mean Lin

Hanzi Lin

Haoran Lin

Qiang Lin

Stuart Linton

Baozhong Liu

Hong Liu

Jin Liu

Shikai Liu

Tianzhong Liu

Wangta Liu

Wenguang Liu

Wen-Sheng Liu

Zhanjiang Liu

Shaojun Liu

Yonghong Liu

Inmaculada Llamas

Chufang Lo

Katie Lotterhos

Wen-Yong Lou

Ying Lu

Jianguo Lu

Zhenming Lv

André Porto M

Hao Ma

Hongyu Ma

Jason Macrander

Angelico Madaro

Yoshiaki Maeda

Emmanouil Malandrakis

João Mano

Zamorano Serrano María Jesús

Anita Marsaioli

Rosa Martínez-Espinosa

Tadashi Maruyama

Akihiko Maruyama

Yusuke Matsuda

Tadashi Matsunaga

Tomomasa Matsuyama

Gordon McDougall

Joaquín Medina

Mohamed Mehiri

Jie Mei

Laurence Meslet-Cladière 
Concetta Messina

Philippe Michaud

Koji Mikami

Cintia Milagre

Cintia Milagre

Wu Min

James Minto

Marta Bou Mira

Norihiko Misawa

Yoichi Miyake

Hideaki Miyashita

Yoshiyuki Miyazaki

Eric Moellering

Thomas Moen

Bidyut R Mohapatra

Francesco Molinari

Antonio Molinaro

Lucia Montenegro

Tetsushi Mori

Masaya Morita

Mohammad Hossein Morowvat

Changkao $\mathrm{Mu}$

Xingjiang Mu

Sergio Munoz

Akio Murakami

Takeshi Naganuma

Hiroaki Nakano

Yutaka Nakashimada

Takeshi Nakayama

Hideki Nakayama

Masayuki Nara

Elodie Nicolau

Donghong Niu

Yasuyuki Nogata

Tetsuko Noguchi

Nakao Nomura

Akihito Ochiai

Wayne O'Connor

Tatsuya Oda

Clément Offret

Yasushi Ogasawara

Tomohisa Ogawa

Takashi Ohshiro

Takao Ojima

Shigeru Okada

Masahiko Okai

Yoshiko Okamura

Nozomu Okino

Tatsufumi Okino

Janet Oldak

Alvaro Takeo Omori

Sandi Orlic

Takashi Osanai
Ana Otero

Ken Overturf

Noriaki Ozaki

Mike Packer

Yniv Palti

Suzan Pantaroto Vasconcellos

Rubens Pasa

Laura Patterson-Fortin

Elina Peltomaa

Katalin Perei

Omaththage P. Perera

José Perez

Ricardo Perez-Martin

Claudia Piccini

Francesco Pietra

Francesc Piferrer

Sanit Piyapattanakorn

Iuliana Popa

Deborah Power

Anne Marie Power

Jianwen Qiu

Rajan Rajan

Sebastian Rakers

Gábor Rákhely

Khanok Ratanakhanokchai

Mohd Ashraf Rathar

Rozenn Ravallec

Karine Rehel

Xianyun Ren

Jae-Sung Rhee

Clint Rhode

Matthew Rise

Esperazna Rivera de Torre

Guillaume Riviere

Steven Roberts

Beatrice Rocher

Pedro N.S. Rodrigues

Liliana Rojo-Arreola

Giovanna Romano

Catherine Roullier

Vassilios Roussis

Ieva Roznere

Josep Ruiz Sanchez

Jean-Marc Sabatier

Sophie Sablé

Reinhard Saborowski

Naotsune Saga

Eric Saillant

Masahiro Sakai

Toshio Sakamoto

Vanvimon Saksmerprome

Mohamed Salem

Romana Santos
Gianfranco Santovito

Elena Sarropoulou

Shunpei Sato

Cornman Scott

Rosaria Scudiero

Masatsugu Seidou

Masashi Sekino

Joseph Selvin

Stefano Serra

Hongwei Shan

Changwei Shao

Christopher Shaw

Qiong Shi

Toshiyuki Shibata

Katsuhiko Shimizu

Chuya Shinzato

Yoshihiro Shiraiwa

Eiichi Shoguchi

Tiago H. Silva

Satoshi Soda

Koji Sode

Jiuzhou Song

Philippe Soudant

Roberto Spurio

Wolfgang Streit

Yoshimasa Sugiura

Zhenghong Sui

Fanyue Sun

Jun xiu Sun

Luyang Sun

Yonghua Sun

Michio Suzuki

Tohru Suzuki

Eiji Suzuki

Iwane Suzuki

Johan Svenson

Toshiyuki Takagi

Shinichi Takaichi

Fumiaki Takakuwa

Hiroaki Takeuchi

Haruko Takeyama

Yutaka Tamaru

Suxu Tan

Atsuko Tanaka

Tsuyoshi Tanaka

Yasuaki Tanaka

John Theodorou

Olivier Thomas

Emma Timmins-Schiffman

Dirk Tischler

Hiroshi Tomoda

Jingou Tong

Maria Touraki 
Antonio Trincone

Yoshinori Tsuji

Mikio Tsuzuki

Toshiki Uji

Valentina Valenzuela-Muñoz

Marc Vandeputte

João Varela

Michael Veeman

Jan Veenstra

Luisa Villamil

Filip Volckaert

Kris R von Schalburg

Toshiyuki Wakimoto

Geoffrey Waldbieser

Aihua Wang

Chunming Wang

Guangyi Wang

Le Wang

Lianyong Wang

Lingling Wang

Qun Wang

Shaolin Wang

Shi Wang

Xianwei Wang

Xiaozhu Wang

Xu Wang

Yongjun Wang

Chenghui Wang

Zhiyong Wang

Wenwen Wang

Anna Wargelius

Shugo Watabe

Fraz Weber
Udo Wehmeier

Dong Wei

Ingrid Weiss

Gary Wessel

Matthias Wiens

Elizabeth Williams

Suzanne Williams

Peter Wilson

Shuke Wu

Xugan Wu

Xiaoqin Xia

Jun Hong Xia

Yamei Xiao

De Xing

Jian Xu

Peng Xu

Tianjun Xu

Wei Xu

Wenteng Xu

Xuewen Xu

Shiqing $\mathrm{Xu}$

Michiaki Yamashita

Yujia Yang

Yingnan Yang

Nina Yasuda

Motoshige Yasuike

Ko Yasumoto

Irina Yermak

Meisheng Yi

Saichiro Yokoyama

Takao Yoshida

Masaki Yoshida

Hiroyuki Yoshikawa
Goro Yoshizaki

Trine Ytrestøyl

Ziniu Yu

Dahui Yu

Zihao Yuan

Kei Yura

Alias Zazali

Qifan Zeng

Zhiqiang Zeng

Roxana Gabriela Zgârian

Aibin Zhan

Guofan Zhang

Jiquan Zhang

Xiaobo Zhang

Xiaohui Zhang

Xiaojun Zhang

Yong Zhang

Yong-An Zhang

Shicui Zhang

Wei Zhang

Yi Zhang

Jianshe Zhang

Zhe Zhao

Huaiping Zheng

Xianhu Zheng

Bingsheng Zhou

Tao Zhou

Xiaoli Zhou

Publisher's Note Springer Nature remains neutral with regard to jurisdictional claims in published maps and institutional affiliations. 